\title{
Single Particle Analysis with Highly Coherent Electron Source from Cold Field Emission Gun
}

\author{
Naoki Hosogi ${ }^{1}$, Takuma Fukumura ${ }^{1}$, Yuko Shimizu ${ }^{1}$, Hirohumi Iijima ${ }^{1}$ \\ 1. EM Business Unit, JEOL Ltd., 3-1-2 Musashino Akishima, Tokyo 196-8558 Japan
}

Cryo transmission electron microscopy (TEM) provides structural information of a specimen close to its natural state without any artifacts due to the specimen preparation process. Recently, the cryo TEM produces exciting results in structural biology by a method of single particle analysis [1]. This progress of the cryo TEM for high data throughput is realized by a highly sensitive and fast image capturing camera made of direct electron sensor and a wealth of easy-to-use automated functions of the camera and a newly developed dedicated cryo microscope. Furthermore, the other capabilities of the microscopes such as highly coherent beam generated by field emission electron gun, less ice contamination by oil-free, clean vacuum environment and etc. also contributed to the method progress. In particular, a remarkable advancement of single particle analysis leads to a number of achievements of near-atomic resolution structural information on biological complexes, are expected to contribute to drug discovery [2]. In the present study, we examined to evaluate the effect to the results by a cold field emission gun (FEG) for cryo TEM work using some biological samples.

First, we confirm the basic performance of cold FEG. Since the energy spread of the emitted electrons from the cold FEG is reduced to be a half of one from Schottky FEG (Fig. 1), and the size of the virtual source is less than $10 \mathrm{~nm}$, resulting an electron beam with high temporal and spatial coherences. With such a beam, cryo TEM image has high contrast due to their high coherence. In the low dose density, the signal-to-noise ratio $(\mathrm{S} / \mathrm{N})$ of image is mostly determined by a statistical noise of electrons, since dose density in cryo TEM is typically several tens of electrons for angstrom square. Namely, the detectable resolution of a sample structure may be determined with the competition between the statistical noise due to low dose and image contrast due to the coherence. It means that cold FEG has possibility to have higher resolution for cryo TEM works.

To compare cold FEG and Schottky FEG, a sample of platinum iridium film was observed with both guns at accelerating voltage of $200 \mathrm{kV}$. Images were taken with a large defocus of $-1000 \mathrm{~nm}$, which is a common in cryo TEM observation. Under this condition, finer lattice was observed in an image obtained with the cold FEG (Fig. 2). We made Fourier-transforms to see the Thon rings from these images. The observed peaks for the highest spatial frequency were corresponding to $0.19 \mathrm{~nm}$ and $0.14 \mathrm{~nm}$ with cold FEG (Fig. 2).

We tried single particle analysis of apoferritin from horse spleen with the cold FEG at $200 \mathrm{kV}$. The structure of apoferritin was resolved with about $3 \AA$ resolution. Fig. 3 shows a slice of $3 \mathrm{D}$ reconstructed structure, which shows the typical structure of the apoferritin. In this slice, spiral structure of $\alpha$-helix can be seen clearly. These results suggest the cold FEG can enhance the image contrast for cryo TEM works.

References:

[1] E Callway, Nature 525 (2015), p. 172.

[2] A Merk et al, Cell 165 (2016), p. 1698. 


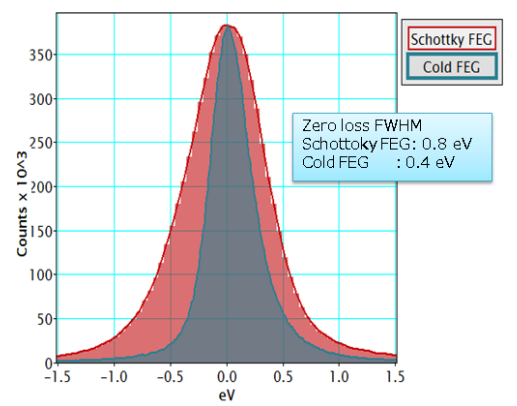

Figure 1. Energy spread of cold FEG and Schottky FEGs. Zero-loss EELS spectra acquired with cold FEG (blue) and Schottky FEG (red). The FWHM (full width at half maximum) of these peaks were measured to be $0.4 \mathrm{eV}$ and $0.8 \mathrm{eV}$, respectively.
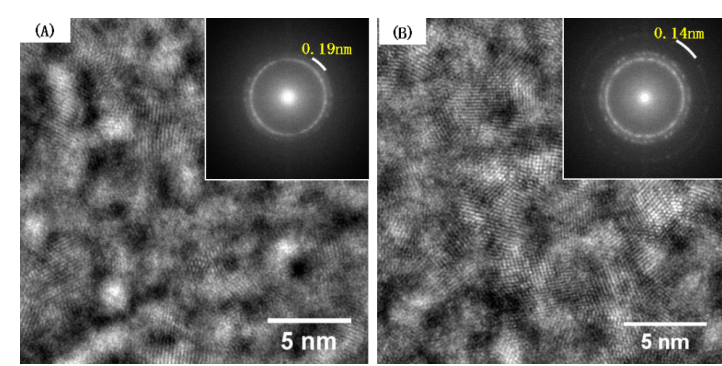

Figure 2. TEM images of platinum iridium acquired with Schottky FEG (A) and cold FEG (B). Both images were captured with a defocus of approximately $-1000 \mathrm{~nm}$. Insets at the upper parts in the both images show Fourier-transformed patterns.

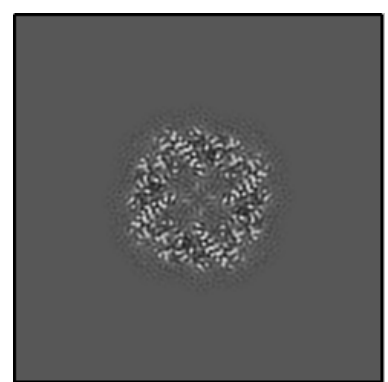

Figure 3. A slice from a 3D reconstructed structure of a apoferritin, which shows the characters of the molecule. 